\title{
The use of Moodle for Formative Assessment in Mathematics
}

Steve Lakin

University of Greenwich

\begin{abstract}
Mathematics is not an easy subject to assess online. However, Moodle has a useful quiz option which can randomly generate questions. In this article, we will investigate the use of multiple-choice quizzes for formative assessment, and discuss their strengths and weaknesses.
\end{abstract}

\section{Introduction}

Technology has become an increasingly large part of modern pedagogy. The Journal of Technology, Pedagogy and Education illustrates current research (Bennett, 2017), while the paper Summative and Formative Assessments in Mathematics Supporting the Goals of the Common Core Standards in the journal Theory Into Practice (Schoenfeld, 2015) is specific to mathematics. Although this article focusses on mathematics and Moodle, the ideas are transferable to other disciplines and platforms.

Mathematics as a subject does not always lend itself well to technology as much as is reliant on pen and paper approaches. However, opportunities are available to utilise technology to enhance the student experience. Moodle is the preferred platform for many universities, including the University of Greenwich. For some courses, it is used primarily as a storage resource, but it also contains powerful tools for online assessment.

Assessment falls into two broad spectrums - formative and summative. While formative assessment is based upon developing a student's understanding, summative assessment is designed to give the student a grade based on the work s/he submits. This article will focus on the formative aspect - we shall look at how to help a student develop their understanding based upon a series of non-assessed online quizzes using the Moodle platform that they are all familiar with.

Mathematics is difficult to assess online summatively.. small interpretative mistakes inadvertent rather than of understanding - by the student can lead to a wrong answer and, often, such misrepresentative errors can be detected only by the assessor's visual scrutiny. Only a sophisticated and thorough mathematical engine underpinning the online assessment process could technologically achieve the same degree of precision - small mistakes can lead to the wrong answer and it is therefore often necessary to use the human eye to detect the mistakes, which is something that cannot be done online without a thorough mathematical engine underpinning the assessment. Nevertheless, multiple choice quizzes can help to develop understanding in a formative sense. Moodle provides the opportunity to do this, allowing for variations on questions. In this article we will discuss how to implement such assessments with variety and provide a critique of its suitability both in mathematics and for general subjects. 


\section{Moodle quizzes}

Moodle has an option to create an online multiple-choice quiz. It was felt that this was most appropriate for formative assessment, as the work involved was prohibitive in scrutinising errors made. Moodle allows for the creation of quizzes derived randomly, both in terms of the order of questions, and the questions themselves.

As an example, running an online quiz twice gave two different questions testing the knowledge of the same concept:

\section{Factorise 418 as a product of primes.}

\section{Select one:}

$2 \times 209$

$1 \times 2 \times 11 \times 19$

$2 \times 11 \times 19$

$22 \times 19$

\section{Factorise 741 as a product of primes.}

\section{Select one:}
$3 \times 247$
$3 \times 13 \times 19$
$39 \times 19$
$1 \times 3 \times 13 \times 19$

Figure 1: Alternative multiple-choice questions to test the same concept 
To perform this randomisation, it is necessary to define variables that are then selected at random; by Moodle. In this particular example, we used the question

\section{Factorise $\{=(\{\mathrm{f}\}+1) *(\{g\} * 2+9) *(2 *\{\mathrm{~h}\}+15)\}$ as a product of primes.}

Here, $\{f\},\{g\}$ and $\{h\}$ represent variables that can be prescribed to be chosen randomly within a given range.

This allows for multiple versions of the same question. The multiple choice options can then be specified, ensuring that one is the correct answer and the other three are variants, including common mistakes that students make - this can be specified in Moodle, such as below:

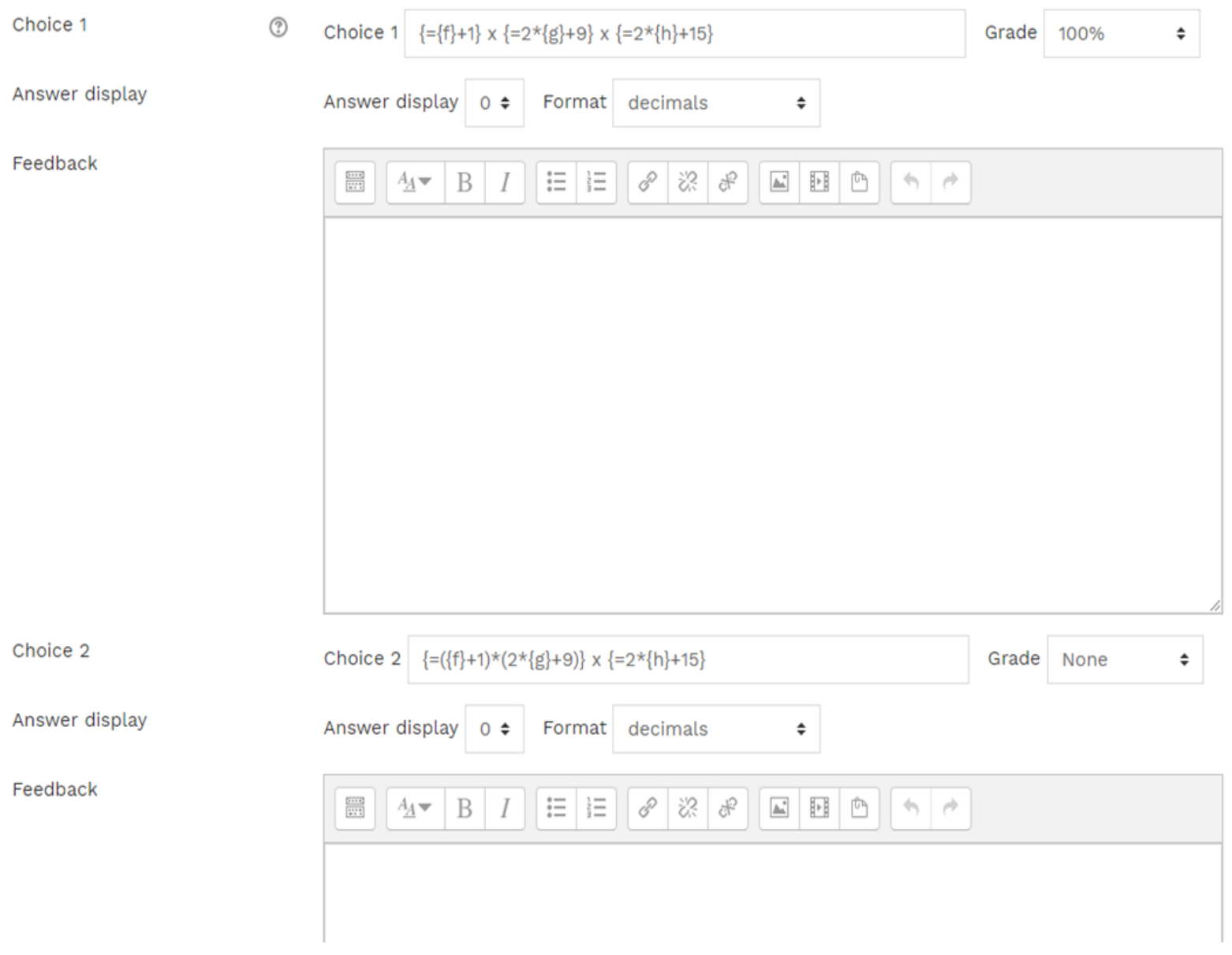

Figure 2: Input of variables to allow for variation

Of course, the precise nature of the question can be varied - this particular version was chosen for simplicity of explanation but can be used for a wide range of applications.

This is undoubtedly a useful application, however it comes with limitations. Moodle does not have many important mathematical functions built in, and it is often necessary to tweak a question to allow for any randomisation. It also does not allow for any graphical randomisation. It is therefore limited but useful. 
For further testing, tools such as Wolfram Alpha (Wolfram Alpha, 2017) are extremely useful. However, students use Moodle on a regular basis and having this option available to them is a valuable resource in developing their formative understanding.

\section{Other subjects}

Although this article has focussed on mathematics, it is clear how this could be applied to subjects with a core mathematical background, such as Engineering.

Hence this is not limited to Mathematics. Many subjects also involve some level of statistics, for which this could be a useful resource. A clear application would be in the Life Sciences where a modelling question could be created with the various parameters chosen randomly.

\section{Feedback}

Following a trial in a Statistics course aimed at Business and Information Technology students, the feedback was overwhelming positive. The primary use of the quizzes for students was that they were very helpful in examination revision, and they found them very useful. In particular, every respondent indicated that it was helpful to have different questions each time they attempted the quiz. There were over ten responses, a sample comment was

\section{"Good - I can repeat and get different questions"}

The material was primarily used for exam revision with around $75 \%$ of respondents stating that this was their primary use.

This was only a limited trial, but the feedback suggested it would be helpful to roll this out into other courses, which is now being done.

It is clear from the student response that this is a useful technique for formative assessment.

\section{Conclusion}

The Moodle quiz system is far from perfect. The limited number of mathematical functions available makes writing suitable questions a challenge. Nevertheless, it has proved to have been successful. It is also an interdisciplinary technique that can be applied to any subject, even though the variation of questions may not be suitable for some subjects.

This is not in any way meant to be a substitute for more formal summative assessment. But as a formative concept, it is a useful tool that can be utilised to help the students gain more understanding, and build confidence as they repeat the tests and progressively obtain a higher score.

\section{Reference list}

Technology, Pedagogy and Teaching

Available at: http://www.tandfonline.com/toc/rtpe20/current (Accessed: 31 October 2017). 


\section{Technology Reviews}

Summative and Formative Assessments in Mathematics Supporting the Goals of the Common Core Standards

Available at: http://www.tandfonline.com/doi/full/10.1080/00405841.2015.1044346

(Accessed: 31 October 2017).

Wolfram Alpha (website) (https://www.wolframalpha.com/, accessed 31 $1^{\text {st }}$ October 2017). 